\title{
Serious Games for Movement Therapy after Stroke
}

\author{
Minhua Ma, Kamal Bechkoum \\ School of Computing \\ Faculty of Business, Computing, and Law \\ University of Derby, Derby, U.K. \\ m.ma@derby.ac.uk
}

\begin{abstract}
This paper details the development and testing of a serious-game based movement therapy aimed at encouraging stroke patients with upper limb motor disorders to practice physical exercises. The system contains a series of Virtual Reality (VR) games. A framework for VR movement therapy is described which consists of a number of serious games designed to encourage patients' physical activity in highly motivating, virtual environments where various factors such as size and gravity can be scaled to adapt to individual patient's abilities and in-game performance. Another goal of this study is to determine whether the provision of serious games based interventions improves motor outcome after stroke. A pilot study with 8 participants who have a first hemispheric stroke shows improvements on impairment measurement and functional measurement shortly after completion of the intervention and 6 weeks after the intervention. Despite its limitations the findings of this study support the effectiveness of serious games in the treatment of participants with hemiplegia. The study also raises awareness of the benefits of using serious games in movement therapy after stroke.
\end{abstract}

Keywords—serious games, Virtual Reality, stroke rehabilitation, movement therapy

\section{INTRODUCTION}

The recent emergence of serious games as a branch of the computer gaming field has introduced the concept of games designed for a serious purpose other than pure entertainment, e.g. healthcare. To date the application of serious games to rehabilitation, such as PlayStation 2's EyeToy and GestureTek's Irex system, has primarily been used as a tool that gives players a novel way to interact with video games in order to promote physical activity. Many studies [1], [2] have identified the benefits of using serious games in movement therapy to improve balance, postural control and fine motor movement. One new mode of movement therapy may be to design and develop an innovative collection of computer games which provide an engaging, self-reinforcing context in which to practice physical activities of the upper limb in people with stroke and to achieve therapeutic goals [3].

This paper details the development and testing of a seriousgame based movement therapy aimed at encouraging stroke patients with upper limb motor disorders to practice physical exercises and therefore enhance endurance, dexterity, speed and range of motion.

\section{A FRAMEWORK FOR VR MOVEMENT THERAPY}

Figure 1 shows our framework for VR movement therapy using functional tasks, such as wrist extension, reaching, grasping and catching, and serious games. The system allows users to interact with virtual objects in real-time through multiple modalities and to practise specific motor skills. Users of the system are stroke patients with motor deficits and physiotherapists who are responsible for initialising the system and controlling the scripting of tasks.

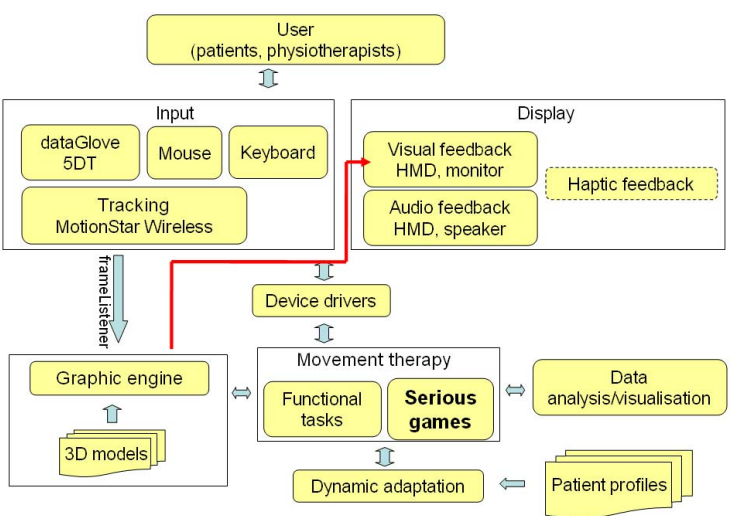

Figure 1. A framework for serious games for movement therapy

Input devices include ordinary devices, mouse and keyboard for the operator and a range of real-time motion tracking devices - two 5DT Ultra DataGloves which capture finger flex and hand postures; Four Ascension MotionStar wireless magnetic sensors are used to track the patient's hand, arm and upper body movements. Output involves visual, audio and haptic modalities. The dual output visual interface includes a desktop computer LCD for the operator and a high resolution HMD, Virtual Research 1280 stereo, for patients. The HMD equipment displays an immersive virtual environment and hence provides a better sense of presence. Haptic interface is a possible extension of the system but it is not our current concern.

The device drivers interface the specialised hardware with the software components which include a 3D graphic engine dealing with loading of mesh data, rendering, scene management and user interface, and a movement therapy 
module which creates functional training and non-functional serious games.

The dynamic adaptation module uses patient profiles and progress data to select tasks and initially configure the difficulty level of the tasks and games. This continues insession, as all patients' interaction with the system is recorded and analysed for dynamic adaptation. Hence the system can provide data on progress for individuals that may be collated across the recovery period or across individuals to provide a database on patient progress. The data analysis module enables visualisation of patients' movement trajectories and displays joint angles, range of movement and velocities.

\section{Functional Training}

The functional training of the system focuses on strengthening exercises of fingers, hands and arms of the paretic limb such as reaching, grasping, placing, and wrist extension. The 3D position and orientation of the user's hand, elbow, and shoulder are sampled via using three magnetic sensors placed on the volar aspect of the hand, the posterior aspect of the elbow, and the anterior aspect of the shoulder. We simulate a virtual kitchen where subjects are asked to manipulate virtual objects of various shapes and sizes at different locations, such as a typical pick-and-place task by reaching and touching a start marker, grasping a virtual cup, and placing it on a saucer some distance away. More extensive discussion of the functional training may be found in [4].

\section{SERIOUS GAMES FOR MOVEMENT THERAPY}

We have developed a number of serious games to encourage patients' physical activity in highly motivating virtual environments where various factors such as size and gravity can be scaled to adapt to individual patient's abilities and in-game performance.

Figure 2 shows the Catch-the-orange game, where the user holds a virtual basket to catch falling oranges which fall at random onto a target area. The position and orientation of the virtual basket are controlled by a sensor attached on a real basket, which the user holds with one hand or both hands (Figure 3B). If the patient tilts the basket, he or she may not able to catch the oranges and the ones already in the basket may fall to the ground. The target area on the $x-z$ plane, the falling speed of oranges (controlled by simulating gravity in the virtual environment), the time between oranges falling and the size of oranges and the basket can all be adjusted to suit individual patient's needs.

Figure 3 shows a fishing game which some participants reported as the most fun and enjoyable game of all. In this game, the player is in a under water world using his hands catch fish which are swimming randomly in the water. The player can see two virtual hands which are representation of his own hands through a sensor attached on each hand (Figure 3B). When both hands collide with a fish, the fish does a little struggling, i.e. wagging its tail drastically on the spot which is implemented by speeding up the speed of swim animation whereas not changing position, and regarded as caught. It then disappears and the number of caught fish is incremented. If only one hand collides with a fish, it will swim away and escape. Since the fish swim in a random manner, if two fish collide with each other, one of them will swim away and the other continue its movement. These are simple AI built in the fish object. The gravity in this under-water virtual environment is also set to zero to free the fish. The size of target area in the 3D space, the size of the fish and their swimming speed, and the size of the virtual hands can all be adjusted to suit individual participant's needs and his in-game performance.

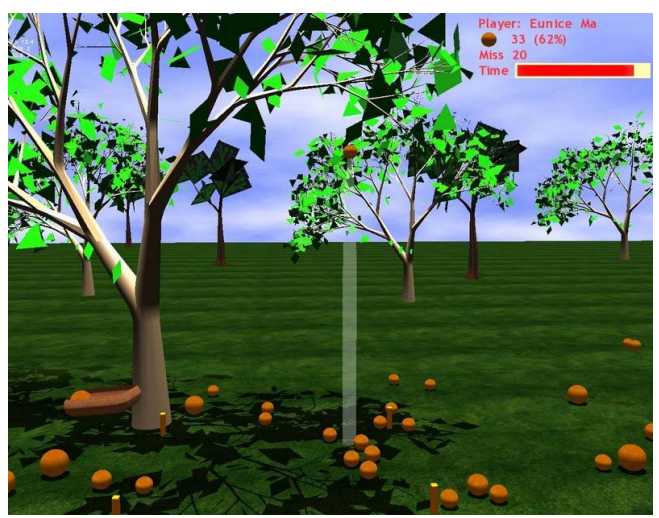

A. A screenshot of the game

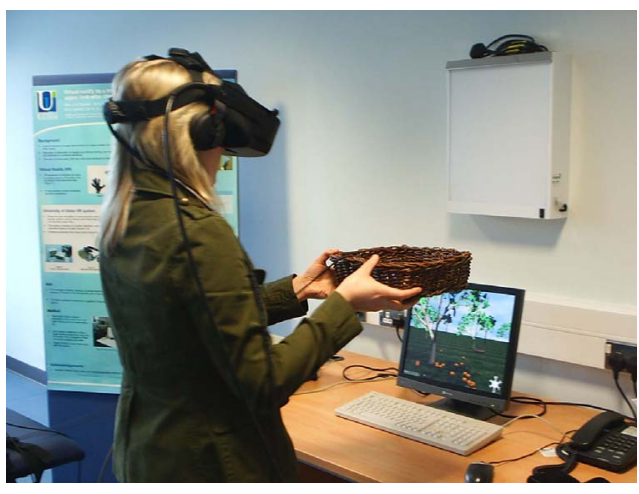

B. A user playing the game

Figure 2. The Catch-the-orange game

Figure 4 shows the whack-a-mouse game. Besides to encourage gross movement and to improve accuracy and speed of users' upper limb movement, the game has also been designed to improve patient's visual discrimination and selective attention, which are important aspects of stroke rehabilitation where the patient exhibits hemi-spatial neglect. The mouse appears at a random location on the table top, stays there for a certain number of seconds (the actual time it stays still depends on the patient profile), and then re-appears on another part of the table. While the mouse is stationary the user tries to hit it using a virtual hammer which is controlled by the position and orientation of a sensor attached on his hand. 


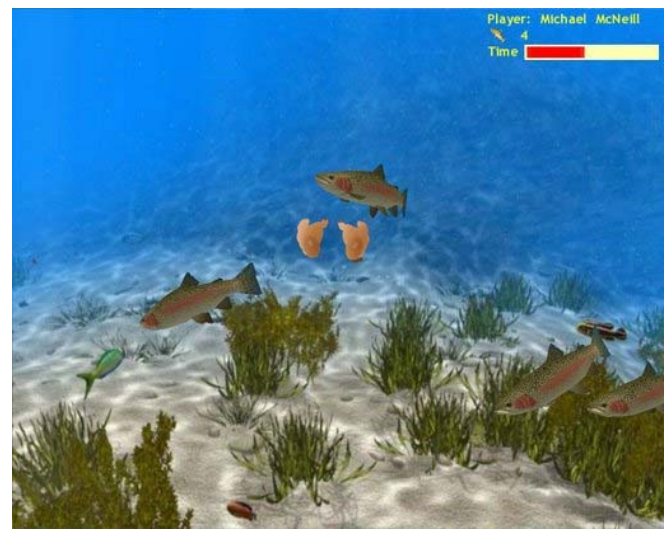

A. A screenshot of the game

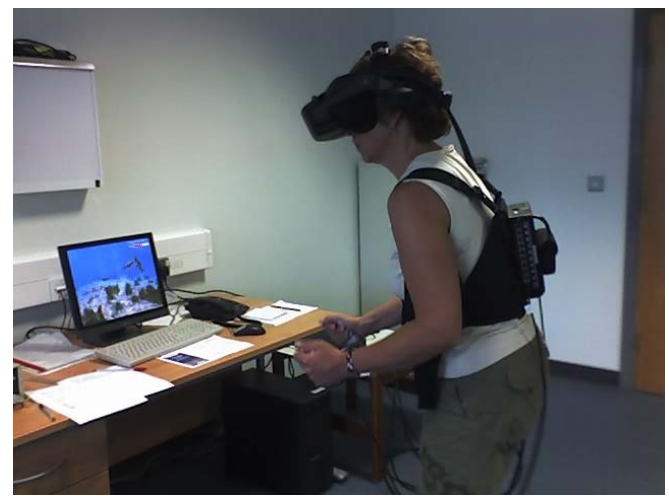

B. A user playing the game

Figure 3 . The fishing game

Initial configuration of the game is done automatically based on the profile of the player. For example, the game will be configured to be easier for patients with serious motor impairment, i.e., the mouse will stay still for a longer period of time for them than for patients with minor motor impairment. The locus of stimuli will also depend on if a) the patient has right or left-sided hemiplegia and b) if the patient exhibits hemi-spatial neglect. For left-sided hemiplegia patients, the random positioning of the mouse is biased towards the right; similarly for the left. If the patient also exhibits hemi-spatial neglect this bias can be increased. It is important that patients are given a chance to familarise themselves with the game and not be presented initially with something that is too difficult, which could lead to disengagement. Therefore we tend to be conservative with the initial setup.

Patients will learn as they play and their progress of learning may be different. Therefore, the initial configuration may no longer be appropriate as the game proceeds. The length of time that the mouse is stationary and the locus of mouse and dog adapt dynamically to patient performance. The mouse appears more frequently in the quadrant where the patient has more misses and seems to have difficulty due to either motor deficit or attention deficit.

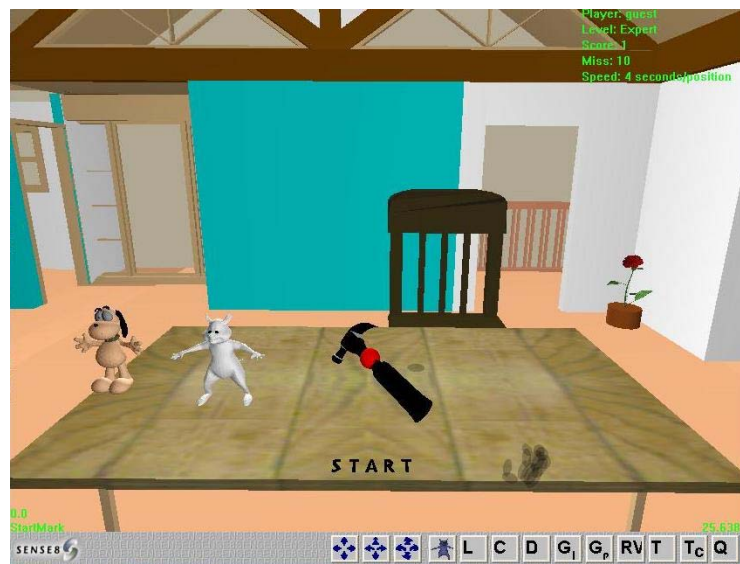

Figure 4. The whack-a-mouse game

There are, therefore, a number of adaptive elements of the game which change dynamically according to how well or badly the user is performing. The game has been programmed to enable automatic progression between three levels (Beginner, Intermediate and Expert) under preset conditions. At the Beginner level the time that the mouse is stationary is set automatically according to the patient profile. At the Intermediate level, the time adapts to the patient's performance (to begin with, the speed is the same as in the beginner level). The game progresses according to a simple accuracy test based on the number of mice hit and the number missed. When the accuracy rate drops below a certain threshold, the length of time that the mouse remains still for increases which has the effect of slowing the game, making it easier; and when the accuracy rate exceeds a certain threshold, this time decreases, making the game harder. The Expert level contains both a mouse and a dog which appear simultaneously: patients must hit as many mice as possible and try to avoid hitting the dog. Hitting a mouse increases the score by one, but hitting a dog decreases it by one. This aspect to the game forces patients to search for and hit only the mouse when both mouse and dog are present, in order to improve visual discrimination and selective attention, which can be important aspect to patient rehabilitation particularly for patients with hemispatial neglect.

The player, level of game, score, and adapted speed are shown in the upper-right corner of the screen to give the user real-time feedback. Overall performance data such as score, accuracy rate, and the length of game-play sessions are also shown to the user when the game is over or on entry to the next level, and this data is also written to a session log file for the user. The next time the patient uses the system this information will be used to configure the game. Further, the physiotherapist can analyse this data to assess how the patient is progressing over a number of sessions. Besides the performance feedback, multiple sensory feedback is given. When a mouse is hit, it squeaks and its colour changes to red, and when a dog is hit, it barks and its colour changes to red. To create an element of competition, but also to give feedback to the patient, the user can view the highest scores of all the users and also his or her personal best scores of each game level. 


\section{EVALUATION}

We user-tested the functional training described in section II.A and the whack-a-mouse games on eight participants who suffer from post-stroke upper limb motor disorders and compared their improvement on impairment and functional measurements.

\section{Participants and Methods}

We have constructed a patient model using traditional symbolic classifiers based on patients' age, time since stroke, left/right hemiplegia, impairment and functional measurement. Table 1 below shows the profile of the 8 patients participating in the study. They were 4 men and 4 women, mean age in years $=56.4 \pm 4.3$ (standard deviation). All participants had a first hemispheric stroke, and all were right handed, i.e. usual handedness. The mean time since stroke until beginning of the intervention was 10.7 months. 4 participants suffer from right hemiplegia and 4 from left hemiplegia.

TABLE 1. USER PROFILES OF THE PARTICIPANTS

\begin{tabular}{|l|l|l|l|l|l|}
\hline $\begin{array}{c}\text { Partici- } \\
\text { pants }\end{array}$ & Age & Gender & $\begin{array}{c}\text { Mths since } \\
\text { stroke }\end{array}$ & $\begin{array}{c}\text { Usual } \\
\text { handedness }\end{array}$ & $\begin{array}{c}\text { L/R } \\
\text { hemiplegia }\end{array}$ \\
\hline S & 49 & Male & 6 & Right & Right \\
\hline J & 49 & Female & 6 & Right & Right \\
\hline F & 66 & Female & 12 & Right & Left \\
\hline R & 74 & Male & 8 & Right & Left \\
\hline P & 45 & Female & 23 & Right & Right \\
\hline B & 75 & Male & 12 & Right & Right \\
\hline E & 69 & Female & 11 & Right & Left \\
\hline C & 43 & Male & 10 & Right & Left \\
\hline
\end{tabular}

Each participant attended ten supervised sessions. Participants S, J, F, R (group A) received both functional training and serious games intervention whereas participants $\mathrm{P}, \mathrm{B}, \mathrm{E}, \mathrm{C}$ (group B) received functional training only. For each patient, three Motricity Index (MI) [5] and Action Research Arm Test (ARAT) [6] scores are measured, representing their status before training, post training, and 6 weeks after training.

\section{Paired t-Test}

Table 2 shows all participants' MI and ARAT scores before, after, and 6 weeks after intervention. Most participants showed improvement in short term, i.e. $\operatorname{MI}(2)-M I(1)$ and ARAT(2)-ARAT(1). However, for some the intervention hasn't shown long-term clinical benefits, i.e. $\mathrm{MI}(3)-\mathrm{MI}(1)$ and ARAT(3)-ARAT(1)

Since our sample sizes are small, we use a paired t-Test on each group and all participants to compare the effect of serious games intervention (Group A) with that of functional training (Group B). In Table 3, the three highlighted t-Test values are the probability associated with participants' MI(1) and MI(2) scores with a one-tailed distribution, if the null hypothesis is true.
TABLE 2. MI AND ARAT SCORES BEFORE, AFTER, AND 6 WEEKS AFTER TRAINING

\begin{tabular}{|l|l|l|l|l|l|l|}
\hline Participants & $\begin{array}{c}\text { MI } \\
\text { (1) }\end{array}$ & $\begin{array}{c}\text { MI } \\
(\mathbf{2})\end{array}$ & $\begin{array}{c}\text { MI } \\
\text { (3) }\end{array}$ & $\begin{array}{c}\text { ARAT } \\
(\mathbf{1})\end{array}$ & $\begin{array}{c}\text { ARAT } \\
(\mathbf{2})\end{array}$ & $\begin{array}{c}\text { ARAT } \\
(3)\end{array}$ \\
\hline Group A & 92 & 100 & 100 & 54 & 54 & 57 \\
\hline S & 73 & 77 & 73 & 45 & 54 & 43 \\
\hline J & 71 & 77 & 71 & 32 & 35 & 35 \\
\hline F & 100 & 100 & 100 & 57 & 57 & 57 \\
\hline R & 77 & 77 & 77 & 57 & 57 & 57 \\
\hline Group B & 77 & 85 & 100 & 54 & 55 & 57 \\
\hline P & 84 & 84 & 84 & 57 & 57 & 57 \\
\hline B & 77 & 79 & 77 & 54 & 53 & 54 \\
\hline E &
\end{tabular}

MI(1) indicates the MI score before training, MI(2) indicates the MI score after training, MI(3) indicates
the MI score 6 weeks after training. Same for ARAT scores.

TABLE 3. PAIRED T-TEST BETWEEN MI(1) AND MI(2)

\begin{tabular}{|l|l|l|}
\hline & \multicolumn{1}{|c|}{ MI(1) } & \multicolumn{1}{|c|}{ MI(2) } \\
\hline S & 92 & 100 \\
\hline J & 73 & 77 \\
\hline F & 71 & 77 \\
\hline R & 100 & 100 \\
\cline { 1 - 3 } Group A t-Test & 0.038997 & \multicolumn{1}{|l}{} \\
\cline { 2 - 3 } & \multicolumn{2}{|l}{}
\end{tabular}

\begin{tabular}{|l|l|l|}
\hline & \multicolumn{1}{|c|}{ MI(1) } & \multicolumn{1}{|c|}{ MI(2) } \\
\hline $\mathrm{P}$ & 77 & 77 \\
\hline $\mathrm{B}$ & 77 & 85 \\
\hline $\mathrm{E}$ & 84 & 84 \\
\hline $\mathrm{C}$ & 77 & 79 \\
\hline Group B t-Test & 0.13916 & \\
\hline
\end{tabular}

\begin{tabular}{|l|l|l|}
\hline & \multicolumn{1}{|c|}{ MI(1) } & MI(2) \\
\hline S & 92 & 100 \\
\hline J & 73 & 77 \\
\hline F & 71 & 77 \\
\hline R & 100 & 100 \\
\hline P & 77 & 77 \\
\hline B & 77 & 85 \\
\hline E & 84 & 84 \\
\hline C & 77 & 79 \\
\hline All participants t-Test & 0.012807 & \multicolumn{1}{|l}{} \\
\cline { 2 - 3 } &
\end{tabular}

The results show that the probability of observing the improvement between $\mathrm{MI}(2)$ and $\mathrm{MI}(1)$ in Group A is 0.038997 , in Group B is 0.13916 , and for all participants is 0.012807 . Therefore, the data support that all participants had significant improvment immediately after intervention (because 0.012807 is a very small probability and the null hypothesis is refuted), and that the intervention on Group A was more effective than the intervention on Group B (because 0.038997 is much smaller than 0.13916). Similar results were observed with the paired t-Test on each group associated with participants' ARAT(1) and ARAT(2) scores. Hence, the 
serious games intervention did have an impact on the recovery of movement.

\section{Case Study on Serious Games Intervention}

Here we have a close look on the two cases participant $\mathrm{J}$ and F closely by analysing their improvements on ARAT and MI scores and game performance in the adaptive whack-amouse game (Table 4 and 5). Both participants' game performance improves significantly over the sessions in terms of their reaction speed, i.e. how many seconds it took them to hit a mouse (see Figure 5A, Figure 6A) and how many seconds a mouse stays still (see Figure 5B, Figure 6B).

TABLE 4. PARTICIPANT J'S GAME PERFORMANCE IN THE WHACK-A-MOUSE GAME

\begin{tabular}{|l|l|l|l|l|l|l|l|}
\hline Date & $\begin{array}{l}\text { Game } \\
\text { level }\end{array}$ & Score & Miss & $\begin{array}{c}\text { Duration } \\
\text { (sec) }\end{array}$ & $\begin{array}{c}\text { Error } \\
\text { rate \% }\end{array}$ & $\begin{array}{c}\text { Speed } \\
\text { (sec/ } \\
\text { mouse) }\end{array}$ & $\begin{array}{l}\text { Interval } \\
\text { (sec) }\end{array}$ \\
\hline $29 / 11 / 06$ & 1 & 15 & 5 & 120 & 25.0 & 8.0 & 6 \\
\hline $04 / 12 / 06$ & 1 & 10 & 5 & 120 & 33.3 & 12.0 & 8 \\
\hline & 1 & 9 & 11 & 120 & 55.0 & 13.3 & 6 \\
\hline & 1 & 15 & 3 & 120 & 16.7 & 8.0 & 7 \\
\hline $13 / 12 / 06$ & 1 & 14 & 4 & 120 & 22.2 & 8.6 & 7 \\
\hline & 2 & 20 & 0 & 120 & 0.0 & 6.0 & 5 \\
\hline & 1 & 21 & 4 & 120 & 16.0 & 5.7 & 5 \\
\hline $15 / 12 / 06$ & 1 & 23 & 2 & 120 & 8.0 & 5.2 & 5 \\
\hline & 2 & 28 & 5 & 120 & 15.2 & 4.3 & 3 \\
\hline & 1 & $\mathbf{4 0}$ & 1 & 120 & 2.4 & 3.0 & 3 \\
\hline & 2 & 38 & 16 & 120 & 29.6 & 3.2 & 2 \\
\hline $20 / 12 / 06$ & 1 & 34 & 6 & 120 & 15.0 & 3.5 & 3 \\
\hline & 2 & 42 & 16 & 120 & 27.6 & 2.9 & 2 \\
\hline & 1 & 36 & 5 & 120 & 12.2 & 3.3 & 3 \\
\hline $15 / 01 / 07$ & 1 & 34 & 7 & 120 & 17.1 & 3.5 & 3 \\
\hline & 2 & $\mathbf{5 0}$ & 21 & 120 & 29.6 & 2.4 & 2 \\
\hline & 3 & $\mathbf{1 3}$ & 16 & 120 & 55.2 & 9.2 & $3-4$ \\
\hline Highest score of Level 1 = 40 & & & & \\
\hline Highest score of Level 2 $=\mathbf{5 0}$ & & & & \\
\hline Highest score of Level 3=13 &
\end{tabular}

The error-rate spike (the highlighted text $55.2 \%$ in Table 4) and speed spike (the highlighted text $9.2 \mathrm{sec} /$ mouse in Table 4) at the end breaking the overall trend of patient $\mathrm{J}$ is due to the fact that in his last session he was able to proceed to level 3 (the expert level) of the game in which both a mouse and a dog appear simultaneously and hitting the dog erroneously will increase the error rate and decrease the score. Having removed this irregular data on level 3, we plot the charts on participant J's speed and interval as shown in Figure 5.

Figure 5A shows the exponential trend of participant J's speed during the sessions, with equation and the square of correlation coefficient $\left(\mathrm{R}^{2}\right)$, and Figure 5B shows the exponential trend of his intervals. Both show a significant improvement on speed of movement and response time.

Participant $\mathrm{F}$ never managed to proceed to the level 3 of the game. Figure $6 \mathrm{~A}$ and $\mathrm{B}$ plot the exponential trends of his speed and interval over his sessions. Though the equation on his speed $\left(\mathrm{y}=14.481 \mathrm{e}^{-0.0706 \mathrm{x}}\right)$ shows a different learning model comparing with participant J's $\left(y=12.13 \mathrm{e}^{-0.103 x}\right)$, significant improvement on both speed and interval was observed too.

Correlating their game performance shown above and their improvement in the real world in term of MI and ARAT scores in Table 2, we find that the computer-based motor therapy using both functional training and serious games was more effective than using functional training solely, in terms of improving motor functions shortly after completion of the intervention and it may bring long term benefits as well. Serious games therefore appear to have potential to become a useful tool for movement therapy.

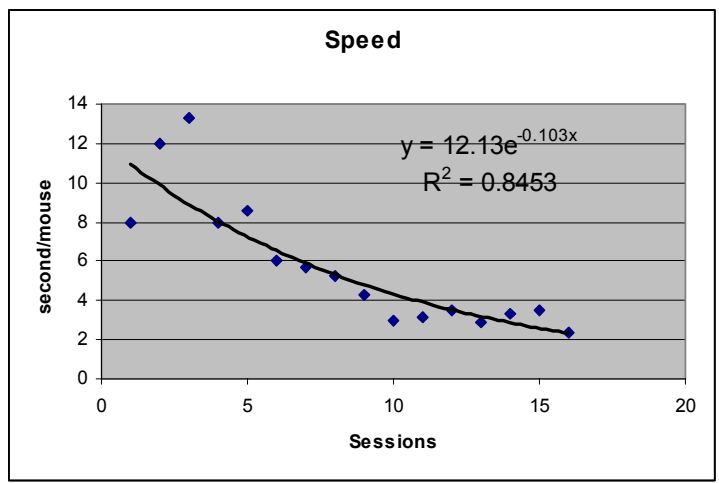

A. Exponential trend of participant J's speed

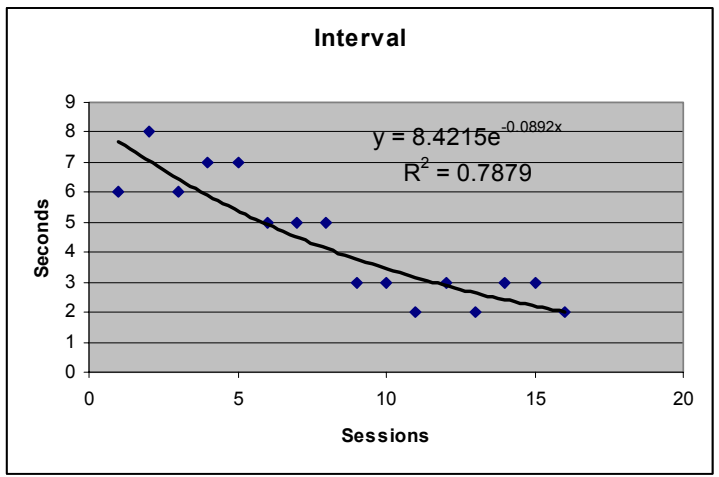

B. Exponential trend of participant J's interval

Figure 5. Participant J's charts

TABLE 5. PARTICIPANT F'S GAME PERFORMANCE IN THE WHACK-A-MOUSE GAME

\begin{tabular}{|l|l|l|l|l|l|l|l|}
\hline Date & $\begin{array}{c}\text { Game } \\
\text { level }\end{array}$ & Score & Miss & $\begin{array}{c}\text { Duration } \\
\text { (sec) }\end{array}$ & $\begin{array}{c}\text { Sror rat } \\
\mathbf{o}\end{array}$ & $\begin{array}{c}\text { Speed } \\
\text { (sec/ } \\
\text { mouse) }\end{array}$ & $\begin{array}{l}\text { Interval } \\
\text { (sec) }\end{array}$ \\
\hline $10 / 10 / 06$ & 1 & 5 & 2 & 60 & 28.6 & 12.0 & 10 \\
\hline $11 / 10 / 06$ & 1 & 4 & 4 & 60 & 50.0 & 15.0 & 8 \\
\hline & 1 & 5 & 3 & 60 & 37.5 & 12.0 & 8 \\
\hline & 1 & 13 & 1 & 120 & 7.1 & 9.2 & 10 \\
\hline $16 / 10 / 06$ & 1 & 12 & 3 & 120 & 20.0 & 10.0 & 8 \\
\hline & 2 & 15 & 2 & 120 & 11.8 & 8.0 & 8 \\
\hline $18 / 10 / 06$ & 1 & 11 & 4 & 120 & 26.7 & 10.9 & 8 \\
\hline & 2 & 15 & 1 & 120 & 6.3 & 8.0 & 8 \\
\hline $23 / 10 / 06$ & 1 & 14 & 4 & 120 & 22.2 & 8.6 & 7 \\
\hline & 2 & 17 & 3 & 120 & 15.0 & 7.1 & 7 \\
\hline $25 / 10 / 06$ & 1 & 16 & 5 & 120 & 23.8 & 7.5 & 6 \\
\hline $30 / 10 / 06$ & 1 & 20 & 5 & 120 & 20.0 & 6.0 & 5 \\
\hline $01 / 11 / 06$ & 1 & 18 & 7 & 120 & 28.0 & 6.7 & 5 \\
\hline
\end{tabular}




\begin{tabular}{|c|c|c|c|c|c|c|c|}
\hline & 2 & 26 & 4 & 120 & 13.3 & 4.6 & 5 \\
\hline $06 / 11 / 06$ & 1 & 26 & 5 & 120 & 16.1 & 4.6 & 4 \\
\hline \multicolumn{5}{|c|}{ Highest score of Level $1=\mathbf{2 6}$} & & & \\
\hline \multicolumn{5}{|c|}{ Highest score of Level $2=\mathbf{2 6}$} & & & \\
\hline
\end{tabular}

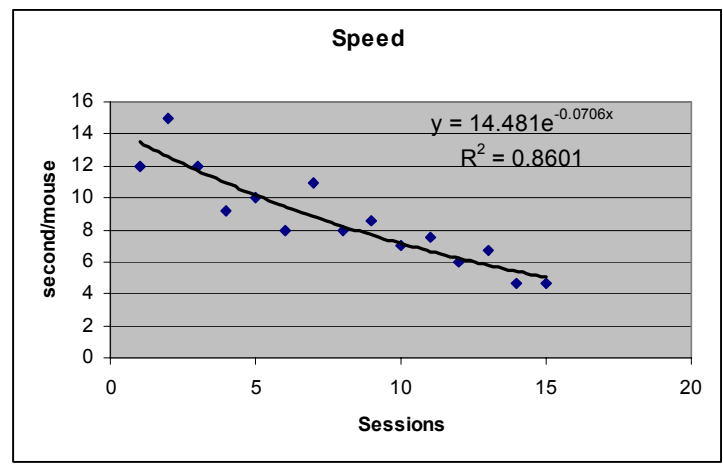

A. Exponential trend of participant F's speed

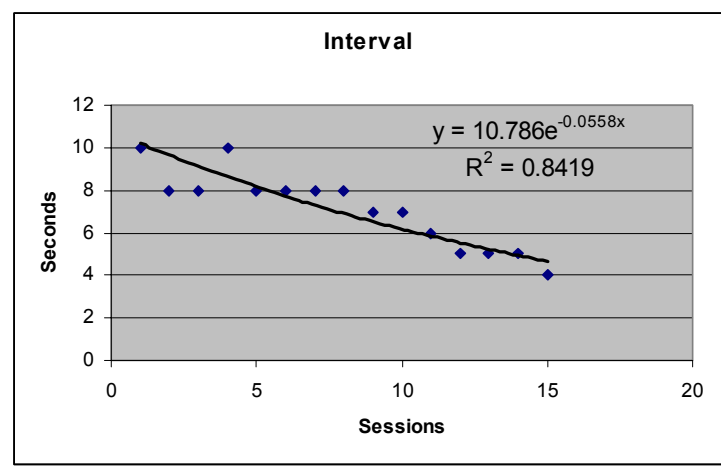

B. Exponential trend of participant F's interval

Figure 6. Participant F's charts

\section{CONCLUSION}

This paper has shown how serious games can contribute to motor therapy by providing motivating tasks. A framework for movement therapy with adaptive game configuration based on data collected before-game and in-game was described which is currently being used by a number of stroke patients with various levels of severity. The integration of VR simulation with serious games adds richness to the virtual environment which has the potential for improving patient outcome. Initial results of user trial are very positive, showing the serious games intervention had an impact on the recovery of movement both in the real world impairment and functional measurements and in-game performance, with patients reporting that they enjoy playing the games. Further work is necessary to compare this methodology with conventional physiotherapy in large sample sizes to determine how much the introduction of serious games actually influences patient outcome.

Serious games intervention may eventually prove to be motivational and beneficial in movement therapy. In conducting this work we hope to raise awareness of the benefits of using serious games in stroke rehabilitation and ultimately improve movement therapy.

\section{ACKNOWLEDGMENT}

This project was supported by the Northern Ireland Chest, Heart and Stroke Association and the Strategic Priority Fund from Department for Employment and Learning. The development and user trial were conducted at University of Ulster. We are grateful for suggestions and guidance on the serious games design from Dr. Michael McNeill of the School of Computing and Information Engineering, University of Ulster. We also would like to thank Jacqui Crosbie for her work on collecting data in clinical trials and Prof. Suzanne McDonough for acquisition of funding. Both of them are in the Health and Rehabilitation Sciences Research Institute, University of Ulster. Finally, our sincere thanks are due to Dr. Stuart Berry of the Derbyshire Business School, University of Derby, for his help on statistical data analysis of the results.

\section{REFERENCES}

[1] Holden, M.K.: Virtual Environments for Motor Rehabilitation: Review CyberPsychology \& Behavior, 8(3): (2005) 187-211.

[2] Fulk, G.D.: Locomotor Training and Virtual Reality-based Balance Training for an Individual with Multiple Sclerosis: A Case Report, Journal of Neurologic Physical Therapy (2005) 29(1):34-42.

[3] Ma, M., M. McNeill, D. Charles, S. McDonough, J. Crosbie, L. Oliver, and C. McGoldrick (2007) Adaptive Virtual Reality Games for Rehabilitation of Motor Disorders. Lecture Notes in Computer Science series. C. Stephanidis (Ed.): Universal Access in Human-Computer Interaction, Part II, HCII 2007, LNCS 4555, 681-690, Springer-Verlag Berlin Heidelberg.

[4] McNeill, M.D.J., Pokluda, L., McDonough, S., and Crosbie, J.: Immersive virtual reality for upper limb rehabilitation following stroke, IEEE International Conference on Systems, Man and Cybernetics, October (2004) 2783-2789.

[5] Demeurisse G., Demol, O., and Robaya E.: Motor evaluation in vascular hemiplegia. European Neurology, 19 (1980) 382-389.

[6] Lyle, R.C.A.: Performance test for assessment of upper limb function in physical rehabilitated treatment and research. Int J Rehabil, 4 (1981) 483-492. 